\title{
Neurotoxicose em bovinos associada ao consumo de bagaço de malte contaminado por Aspergillus clavatus ${ }^{1}$
}

\author{
Pedro Soares Bezerra Jr²*, Djeison Lutier Raymundo ${ }^{3}$, Andréia Spanamberg ${ }^{4}$, \\ André Mendes Ribeiro Corrêa ${ }^{3}$, Jorge J. Bangel Júnior ${ }^{5}$, Laerte Ferreiro ${ }^{4}$ e \\ David Driemeier ${ }^{3}$
}

\begin{abstract}
Bezerra Jr P.S., Raymundo D.L., Spanamberg A., Corrêa A.M.R., Bangel Jr J.J., Ferreiro L. \& Driemeier D. 2009. [Neurotoxicosis in cattle associated with consumption of beer residues contaminated with Aspergillus clavatus.] Neurotoxicose em bovinos associada ao consumo de bagaço de malte contaminado por AspergiIlus clavatus. Pesquisa Veterinária Brasileira 29(3):220-228. Setor de Patologia Veterinária, Universidade Federal do Rio Grande do Sul, Av. Bento Gonçalves 9090, Porto Alegre, RS 91540-000, Brazil. E-mail: bezerraj@ufla.br

Two outbreaks of a neurological disease affecting herds of dairy cattle that were fed moldy beer residues contaminated with Aspergillus clavatus in the county of Viamão, Rio Grande do Sul, Brazil, are described. The morbidity of both outbreaks was $30 \%$ and the lethality $50 \%$ and $100 \%$. The clinical course varied from 5 to 64 days. Only one of the animals that recovered from the disease remained with slight locomotor sequels. Clinical signs were predominantly locomotor and included muscle tremors of varied intensity, hyperesthesia and progressive posterior ataxia, paresis and paralysis with knuckling of fetlocks of the hind limbs. Gait abnormalities were more pronounced after exercises which in general led to falling down. There was also reduced milk production, but appetite and water intake were maintained until close to death or euthanasia. From five cattle necropsied, two showed macroscopic lesions characterized by necrotic changes and mineralization in pelvic muscles and thoracic limbs. The main histological findings consisted of chromatolytic neuronal degeneration and necrosis in selected nuclei of the brain stem, the ventral horn of the spinal cord, and of the trigeminal, stellate celiac and spinal ganglions. In two cattle there was wallerian degeneration in dorsal funiculi of the spinal cord and ischiadic and fibular nerves. The diagnosis was based on epidemiological data, clinical signs, necropsy findings, histological lesions and mycological examination. Epidemiologic, clinical and pathologic aspects, pathogenetic mechanisms and differential diagnoses are discussed.
\end{abstract}

INDEX TERMS: Aspergillus clavatus, cattle, beer residues, neurotoxicosis, chromatolysis neuronal.

RESUMO.- Descrevem-se dois surtos de uma doença neurológica que afetou rebanhos bovinos leiteiros que consumiam bagaço de malte contaminado por Aspergi-

\footnotetext{
${ }^{1}$ Recebido em 23 de julho de 2008.

Aceito para publicação em 4 de outubro de 2008.

Parte da Tese de Doutorado do primeiro autor, no Curso de Ciências Veterinárias, Universidade Federal do Rio Grande do Sul (UFRGS).

${ }^{2}$ Setor de Patologia Veterinária, Universidade Federal de Lavras (UFLA), Lavras, MG 37200-000, Brasil. *Autor para correspondência: bezerraj@ufla.br

${ }^{3}$ Setor de Patologia Veterinária, UFRGS, Av. Bento Gonçalves 9090, Agronomia, Porto Alegre, RS 91540-000, Brasil.

${ }^{4}$ Laboratório de Micologia, Faculdade de Veterinária, UFRGS, Porto Alegre, RS.

${ }^{5}$ Departamento de Medicina Animal, UFRGS, Porto Alegre, RS.
}

Ilus clavatus no município de Viamão, estado do Rio Grande do Sul. A morbidade em ambos os surtos foi em torno de $30 \%$ e a letalidade, $50 \%$ e $100 \%$. A evolução clínica da doença variou de 5 a 64 dias. Dentre os animais que se recuperaram apenas um permaneceu com seqüelas locomotoras leves. Os sinais clínicos eram predominantemente locomotores e incluíam tremores musculares de intensidade variável, hiperestesia e membros pélvicos com ataxia, paresia e paralisia progessivas, e apoio sobre os boletos. Os distúrbios locomotores eram intensificados pelo exercício que, em geral, desencadeava quedas. Havia também marcada queda na produção leiteira, no entanto o apetite e a dipsia eram mantidos até próximo da morte ou eutanásia. Cinco bovinos foram necropsiados e 
destes dois apresentaram lesões macroscópicas nos músculos esqueléticos, principalmente nos membros pélvicos e torácicos caracterizadas por alterações necróticas e mineralização. No sistema nervoso, os principais achados consistiam de degeneração e necrose neuronal cromatolítica em núcleos nervosos específicos no tronco encefálico, nos cornos ventrais da medula espinhal e nos gânglios trigeminal, estrelado, celíaco e espinhais. Em dois bovinos havia adicionalmente degeneração walleriana nos funículos dorsais da medula espinhal e nervos isquiádico e fibular. O diagnóstico foi baseado nos dados epidemiológicos, sinais clínicos, achados de necropsia, histopatológicos e micológicos. Os aspectos epidemiológicos, clínicos e patológicos da enfermidade, além de possíveis mecanismos patogenéticos e diagnósticos diferenciais são discutidos.

TERMOS DE INDEXAÇÃO: Aspergillus clavatus, bovinos, bagaço de malte, neurotoxicose, cromatólise neuronal.

\section{INTRODUÇÃO}

O bagaço de malte é um subproduto do processo de produção de cervejarias formado pela parte sólida obtida da filtração do mosto cervejeiro antes da fervura. Este é constituído principalmente de restos de casca e polpa de cevada, mas também dos grãos do adjunto, como arroz, milho e trigo. $O$ bagaço de malte é quantitativamente 0 principal subproduto do processo cervejeiro, sendo gerado de $14-20 \mathrm{~kg}$ a cada 100 litros de cerveja produzida. A grande produção anual de cerveja no país, em média 8,5 bilhões de litros, dá uma idéia da enorme quantidade deste subproduto gerada (Santos \& Ribeiro 2005). Em razão de suas características nutricionais, grande disponibilidade e menor custo este subproduto vem sendo amplamente utilizado na alimentação de bovinos com aptidão leiteira no Rio Grande do Sul (Barros et al. 2006) e outras regiões do país (Santos \& Ribeiro 2005).

O consumo de subprodutos de cervejarias ou destilarias contaminados pelo fungo Aspergillus clavatus tem sido associado a uma doença neurológica em bovinos e ovinos em diversos países (Kellerman et al. 1976, 1984, Gilmour et al. 1989, Sabater-Vilar et al. 2004, Kellerman et al. 2005). No Brasil o diagnóstico desta enfermidade com lesões características foi feito por Loretti et al. (2003).

A. clavatus é um fungo saprófito cosmopolita normalmente isolado de amostras de solo, fezes e materiais vegetais em decomposição. Embora considerado um contaminante relativamente raro, principalmente de cereais e seus produtos, havendo condições favoráveis para o seu crescimento e produção de toxinas, o fungo pode proliferar e predominar sobre outros microorganismos. Isso tende a ocorrer em produtos estocados com alto teor de umidade (Lopez-Dias \& Flannigan 1997, Varga et al. 2003) e particularmente em temperaturas superiores a $13^{\circ} \mathrm{C}, \mathrm{com}$ intenso crescimento a $25^{\circ} \mathrm{C}$ (Lopez-Dias \& Flannigan 1997).

A natureza da(s) micotoxina(s) envolvida(s) na síndrome tóxica relacionada a $A$. clavatus ainda não é bem esclarecida (Lopez-Dias \& Flannigan 1997, McKenzie et al. 2004, Sabater-Vilar et al. 2004). O fungo tem sido apontado como produtor de diversas micotoxinas, como patulina, brefeldina A, citocalasina E e K, ascladiol, clavatol, ácido cójico e diversas substâncias tremorgênicas, como triptoquivalona, nortriptoquivalona, triptoquivalina e gliantripina (Kellerman et al. 1976, 1988, Varga et al. 2003, Sabater-Vilar et al. 2004). Entretanto, nenhuma destas foi testada em ruminantes para determinar sua participação na patogênese da doença (McKenzie et al. 2004). Alguns autores, no entanto, especulam que a enfermidade seja resultado da ação sinérgica de várias micotoxinas (Varga et al. 2003).

À necropsia, animais que sobrevivem por longos períodos, podem apresentar áreas pálidas de degeneração e necrose nos músculos esqueléticos dos membros pélvicos e torácicos, e em alguns casos, também no coração (Kellerman et al. 1976, 2005). Microscopicamente, há degeneração e necrose de grandes neurônios, especialmente dos cornos ventrais da medula espinhal, núcleos específicos da medula oblonga, mesencéfalo e tálamo, e gânglios espinhais (Gilmour et al. 1989, Van der Lugt et al. 1994, Kellerman et al. 2005). Degeneração axonal central e periférica com perda secundária de mielina tem sido identificada em alguns surtos (Gilmour et al. 1989, Van der Lugt et al. 1994). Histologicamente, as lesões nos músculos esqueléticos e cardíacos se apresentam na forma de degeneração e necrose hialina e flocular acompanhadas ou não de mineralização (Kellerman et al. 1976, Loretti et al. 2003). A patogênese das lesões permanece controversa. Tem sido sugerido que a cromatólise neuronal seja uma manifestação de injúria primária ao corpo do neurônio. Neste sentido, a degeneração axonal, observada em alguns casos, seria secundária ou concomitante (Kellerman et al. 1976, 1984, Van der Lugt et al. 2002). Porém alguns autores propõem que as lesões podem ser explicadas por uma injúria axonal primária e que as alterações observadas em corpos de neurônio sejam secundárias e definidas como reação axonal (Gilmour et al. 1989, Van der Lugt et al. 1994).

O presente trabalho tem como objetivo descrever os aspectos clínicos e patológicos de dois surtos de uma doença neurológica em bovinos consumindo bagaço de malte contaminado por $A$. clavatus. Os aspectos epidemiológicos, clínicos e patológicos da enfermidade, além de possíveis mecanismos patogenéticos e diagnósticos diferenciais serão discutidos.

\section{MATERIAL E MÉTODOS}

Dados epidemiológicos e clínicos dos surtos foram obtidos em visitas às propriedades e junto aos proprietários. Na Propriedade 1 foram necropsiados três bovinos, um já em avançado estado de autólise e dois após eutanásia com tiopental sódico quando moribundos. Na Propriedade 2 foram necropsiados dois bovinos, um imediatamente após a morte e outro após eutanásia com tiopental sódico quando moribundo.

Os fragmentos de diversos tecidos destinados à microscopia óptica foram fixados em formol a $10 \%$ tamponado, desidratados 
em concentrações crescentes de álcool etílico, diafanizados em xilol e incluídos em parafina. Cortes de $5 \mathrm{~mm}$ de espessura foram corados pela hematoxilina e eosina e pelos métodos de violeta de cresil e azul rápido de luxol (Prophet et al. 1992). Do sistema nervoso dos animais foram coletados encéfalo, medula espinhal, gânglios nervosos (trigeminal, espinhal, celíaco e estrelado), fragmentos dos nervos isquiádico e fibular. Amostras do sistema nervoso central incluíram cortes da medula oblonga ao nível do óbex, ponte com pedúnculos cerebelares (ao nível do sulco bulbo-pontino), córtex cerebelar (ao nível do corte anterior), mesencéfalo ao nível dos colículos rostrais, tálamo caudal (secção caudal ao corpo mamilar) e cranial (ao nível do quiasma óptico), córtex cerebral nos lobos occipital (ao nível do corte mais caudal do tálamo) e frontal (na altura do sulco cruzado), e medula espinhal ao nível torácico e das intumescências lombar e cervical. A classificação dos núcleos nervosos com lesões seguiu a descrição neuroanatômica de Yoshikawa (1968). Amostras de músculos dos membros pélvicos (semitendinoso, vasto lateral, vasto medial e gastrocnêmio), membros torácicos (tríceps braquial e subescapular), axiais (longíssimo dorsal e psoas), respiratórios (intercostal e diafragma) e cardíacos (ventrículos e septo interventricular) dos animais foram mantidas em geladeira $\left(4^{\circ} \mathrm{C}\right)$ por cerca de $24-48$ horas antes da fixação em formol para evitar artefatos de contração. Foram avaliados ainda fragmentos dos seguintes órgãos: pulmão, fígado, rim, pâncreas, adrenal, tireóide, hipófise, baço, linfonodos, rúmen, retículo, omaso, abomaso, intestinos e bexiga.

Cortes selecionados do sistema nervoso foram submetidos à imunoistoquímica através do método da steptavidina-biotina marcada (LSAB). Foram utilizados os seguintes anticorpos primários: a) anti-proteína fibrilar glial ácida (anti-GFAP), b) policlonal de coelho anti-neurofilamento bovino $\mathrm{H}$ de $200 \mathrm{kD}$ (Serotec: AHP245), c) monoclonal anti-proteína príon (VRMD: F99/97).

Amostras do bagaço de malte utilizado nas propriedades que apresentavam visível contaminação fúngica foram coletadas e enviadas para identificação e cultivo micológico no Laboratório de Micologia Veterinária da Universidade Federal do Rio Grande do Sul. Micélios e conídios foram removidos destas amostras, montados em lactofenol para exame microscópico e inoculados em placas de Petri contendo Agar Sabouraud Dextrose (OXOID) acrescido de $0,4 \mathrm{~g}$ de cloranfenicol (INLAB). A incubação foi realizada num período mínimo de 10 dias a uma temperatura de $25-30^{\circ} \mathrm{C}$. Posteriormente os isolados identificados como Aspergillus clavatus foram repicados através de transferência de micélios e conídios em Agar Sabouraud para obtenção de cultura pura que foi armazenada em água destilada estéril (técnica de Castellani).

Para pesquisa de patulina foram enviadas ao Laboratório de Análises Micotoxicológicas (Lamic) da Universidade Federal de Santa Maria (UFSM) amostras do bagaço de malte utilizado na Propriedade 1. A pesquisa de patulina foi realizada por cromatografia líquida de alta eficiência, com detecção por ultravioleta (HPLC/UV). Os dados meteorológicos foram obtidos em consulta ao Instituto Nacional de Meteorologia (INMET) (www.inmet.com.br).

\section{RESULTADOS}

Os surtos ocorreram entre março de 2006 (Propriedade 1) e fevereiro de 2007 (Propriedade 2), nos bairros de Branquinha e Itapuã do município de Viamão, Rio Grande do Sul, respectivamente. Na Propriedade 1 os sinais clínicos foram observados cerca de uma semana após o recebimento de nova carga de bagaço de malte, que foi colocada sobre as sobras da carga anterior, recebida 28 dias antes. Os animais passaram a receber um mistura de ambas. O proprietário notou surgimento de um bolor esverdeado ou acinzentado sobre o bagaço de malte. Nesse bolor foi identificado e isolado Aspergillus clavatus. O uso do subproduto foi suspenso cerca de três dias após serem notados os primeiros sinais clínicos nos bovinos. No entanto, um animal ainda adoeceu cerca de dez dias após a suspensão. Na propriedade eram utilizados cerca de $25 \mathrm{~kg}$ de bagaço de malte por animal por dia. Duas das três vacas que adoeceram eram das de melhor produção (cerca de $40 \mathrm{~kg}$ de leite/dia).

O bagaço de malte utilizado na Propriedade 2 apresentou, segundo o proprietário, um bolor azul esverdeado na superfície 4-5 dias após a entrega. Os primeiros sinais clínicos no primeiro animal afetado foram percebidos 12 dias após entrega do material, ou seja, cerca de 7 dias após a observação do bolor. Esta carga do subproduto foi empregada na alimentação dos animais até 28 dias após entrega. Uma nova carga do subproduto foi recebida no dia seguinte ao término e colocada no mesmo reservatório, apresentando um bolor semelhante ao descrito em poucos dias. Cinco dias após a entrega desta, durante a primeira visita a propriedade, praticamente toda a superfície do subproduto estava recoberta pelo bolor azul esverdeado com pequenas áreas brancas e pretas. Do bolor foi identificado e isolado $A$. clavatus (Fig.1) com pequena contaminação por Rhizopus sp. O uso do subproduto havia sido suspenso no dia anterior à visita. Assim os animais ingeriram o subproduto por cerca de 28 dias após a observação do bolor (24 dias da primeira car-

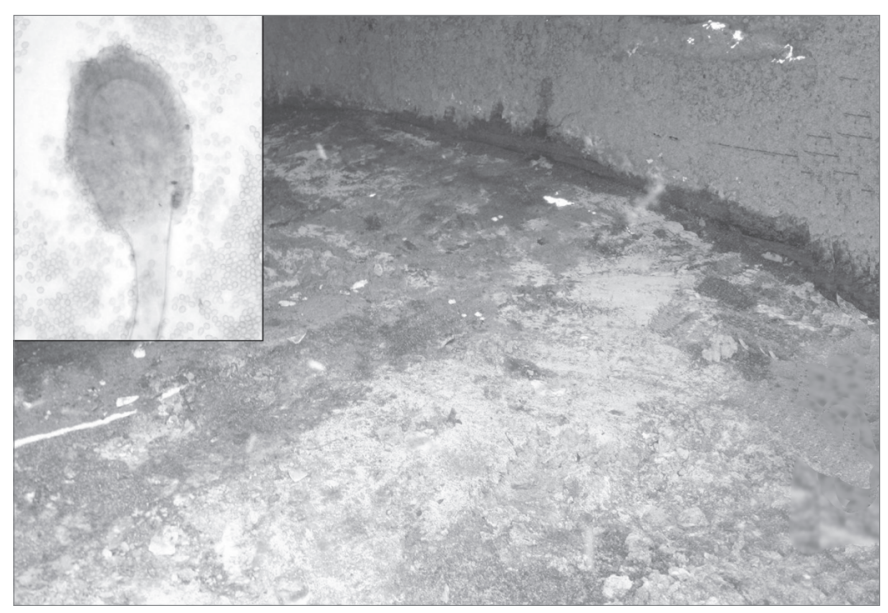

Fig.1. Neurotoxicose causada por Aspergillus clavatus em bovinos. Reservatório do bagaço de malte da Propriedade 2; há abundante bolor azul esverdeado na superfície do subproduto. No detalhe, aspectos microscópicos de $A$. clavatus, vesícula em forma clava recoberta por fiálides curtos (azul mais intenso) e grande número de conídios aderidos às fiálides ou destacados. Lactofenol azul de algodão, obj.100x. 
Quadro 1. Neurotoxicose associada à ingestão de bagaço de malte contaminado por Aspergillus clavatus. Dados dos bovinos dos surtos, com evolução clínica e desfecho

\begin{tabular}{ccc}
\hline Animais Dados dos animais & $\begin{array}{c}\text { Evolução } \\
\text { clínica }\end{array}$ & Desfecho \\
\hline
\end{tabular}

Propriedade 1

Bovino $1 \mathrm{HPB}$ a , fêmea, 7 anos $\mathrm{NI}^{\mathrm{b}}$ Morte com necropsia

Bovino 2 HPB, fêmea, 5 anos 16 dias Eutanásia e necropsia

Bovino 3 HPB, fêmea, 4 anos \pm 10 dias Eutanásia e necropsia

Propriedade 2

Bovino 4 HPB, fêmea, 5 anos 5 dias Morte com necropsia

Bovino 5 HPB, macho, 1,3 ano 22dias Eutanásia e necropsia

Bovino 6 HPB, macho, 8 meses NI Adoeceu levemente e recuperou-se

Bovino 7 HPB, fêmea, 2,5 anos 64 dias Morte sem necropsia

Bovino 8 HPB, fêmea, 2 anos 5 dias Morte sem necropsia

Bovino 9 HPB, fêmea, 2 anos 133 dias Adoeceu moderadamente e seqüelas leves ${ }^{d}$

Bovino $10 \mathrm{HPB}$, macho, terneiro 5 dias Morte sem necropsia

Bovino 11 HPB, fêmea, 2 anos 6 dias $^{C}$ Adoeceu levemente e recuperou-se

Bovino 12 HPB, fêmea, 2 anos 6 dias $^{c}$ Adoeceu levemente e recuperou-se

Bovino $13 \mathrm{HPB}$, fêmea, 2,5 anos 6 dias $^{\mathrm{c}}$ Adoeceu levemente e recuperou-se

a HPB: holandesa preta e branca; ${ }^{\mathrm{b}} \mathrm{NI}$ : não informado; ${ }^{\mathrm{c}}$ Observação de sinais em visita a propriedade, porém sem sinais clínicos aparentes na última visita (117 dias após início dos sinais); ${ }^{\mathrm{d}}$ Morte por distocia quando apresentava seqüelas leves dos sinais locomotores, não necropsiado.

ga e 4 dias da segunda carga) e cerca de 21 dias após os primeiros sinais clínicos. Eram utilizados cerca de $8 \mathrm{~kg}$ de bagaço de malte por animal por dia e não ocorreram novos casos após a suspensão do uso. Há um ano o subproduto era empregado na alimentação do rebanho sem que fossem notados problemas.

A morbidade de ambos os surtos foi semelhante em torno de $30 \%$. Na Propriedade 1 de um total de 10 bovinos que recebiam o bagaço de malte 3 adoeceram (30\%), enquanto que na Propriedade 2 de 33 bovinos, 10 adoeceram $(30,3 \%)$. A letalidade na Propriedade 1 foi de $100 \%$, enquanto que na Propriedade 2 foi de $50 \%$. Os dados dos bovinos dos surtos, assim como evolução e desfecho, são apresentados no Quadro 1. A evolução clínica variou de 10-16 dias (Propriedade 1) e 5-64 dias (Propriedade 2). Dos animais que recuperaram na Propriedade 2 apenas em um, que apresentou sinais mais graves, havia seqüelas locomotores leves até 133 dias após o início dos sinais. Os demais não apresentavam sinais perceptíveis 117 dias após o início dos sinais.

Os sinais clínicos em ambos os surtos eram predominantemente locomotores e incluíam mais comumente fraqueza muscular e incoordenação principalmente dos membros pélvicos, tremores musculares nos flancos e membros, e relutância em se movimentar. Inicialmente os sinais não eram muito perceptíveis se os animais estavam em repouso. No entanto, quando movimentados os tremores musculares e fraqueza muscular tornavam-se mais evidentes. Os tremores eram mais acentuados nos bovi- nos da Propriedade 1. Com a evolução havia extrema dificuldade de permanecer em estação, permanecendo poucos momentos com os membros pélvicos afastados e deslocados cranialmente e os membros torácicos próximos, deslocados caudalmente (Fig.2A). Os bovinos quando forçados a caminhar demonstravam fraqueza muscular, incoordenação e hipermetria predominantemente nos membros pélvicos. Com freqüência o caminhar era trôpego e apoiado sobre os boletos dos membros pélvicos (Fig. 2B), com quedas ocasionais, após as quais estes relutavam por alguns minutos em sair do decúbito esternal. Nos bovinos que evoluíram para o óbito ou foram eutanasiados, esses quadros clínicos progrediam para decúbito esternal permanente, embora permanecessem alertas, com apetite e dipsia relativamente normais até próximo da morte ou eutanásia. Nas fases terminais os bovinos permaneciam em decúbito lateral, com movimentos de pedalagem e em poucos casos opistótono. Os bovinos, especialmente os da Propriedade 2, apresentavam ainda hiperestesia demonstrada por resposta exagerada a estímulos externos, como

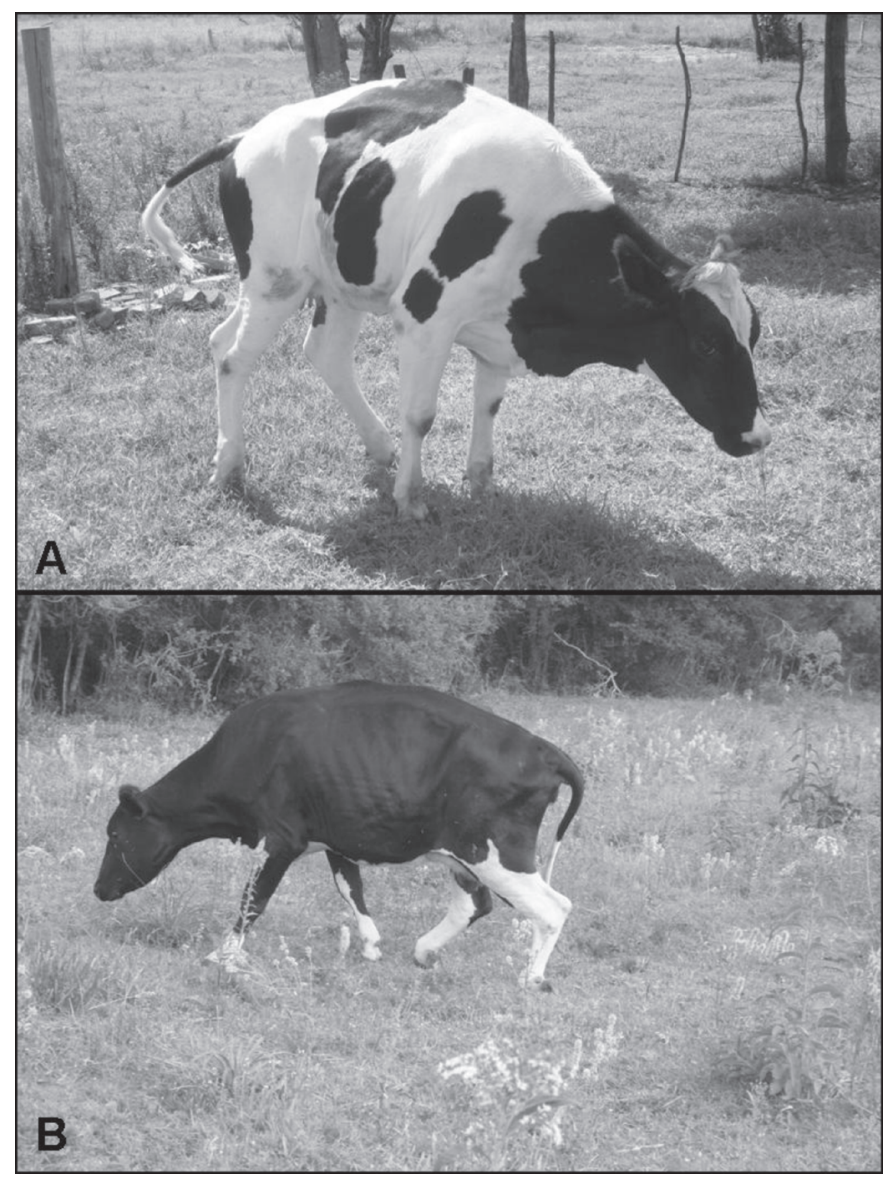

Fig.2. Neurotoxicose causada por Aspergillus clavatus em bovinos. (A) Bovino 9, com membros torácicos próximos e deslocados caudalmente e membros pélvicos afastados e deslocados cranialmente, indicando deslocamento do apoio para os membros torácicos. (B) Bovino 3, caminhando com membros pélvicos parcialmente flexionados e apoiados sobre os boletos. 
toque e estímulos sonoros, e eram mais irritadiços. O Bovino 2 demonstrou adicionalmente clara relutância em transpor pequenos obstáculos e intensificação dos tremores quando em decúbito lateral. O Bovino 9 apresentou ainda moderada taquipnéia após breve movimentação. Quando testada, a sensibilidade cutânea estava diminuída ou ausente principalmente nas porções posteriores do corpo (Bovinos 2, 3 e 4). Foi relatada também em ambas propriedades caquexia nos animais de evolução mais longa e marcada queda na produção dos animais em lactação (na Propriedade 2, onde foi mensurada, a queda chegou a $47,2 \%$ no auge do surto).

As lesões macroscópicas, quando presentes, foram observadas nos músculos esqueléticos dos membros pélvicos e torácicos. No Bovino 2 estas estavam claramente presentes e se caracterizavam por áreas esbranquiçadas focalmente extensas e arenosas com freqüência próximas a inserções dos músculos, contrastando com áreas de coloração normal vizinhas. Nos membros pélvicos estas alterações foram observadas nos músculos quadríceps, semitendinoso, gastrocnêmio, sóleo e fibular terceiro. Já nos membros torácicos, nos músculos tríceps braquial e subescapular. Adicionalmente, havia leve edema gelatinoso e translúcido entre as fáscias musculares. No Bovino 3 havia apenas palidez difusa e leve em músculos dos membros pélvicos e nos Bovinos 1, 4 e 5 não foram observadas lesões macroscópicas significativas.

\section{Quadro 2. Neurotoxicose associada à ingestão de bagaço de malte contaminado por Aspergillus clavatus. Áreas e estruturas do sistema nervoso com lesões microscópicas nos bovinos necropsiados e suas respectivas intensidades $^{a}$}

Áreas ou estruturas do sistema Bovino 2 Bovino 3 Bovino 4 Bovino 5 nervoso com lesões

\begin{tabular}{lcccc}
\hline Intumescência lombar & & & & \\
$\quad$ Corno ventral & ++ & ++ & + & + \\
$\quad$ Corno dorsal & + & + & + & 0 \\
Intumescência cervical & & & & \\
$\quad$ Corno ventral & 0 & ++ & ++ & ++ \\
$\quad$ Corno dorsal & 0 & 0 & 0 & 0 \\
Medula torácica & & & & \\
Corno ventral & 0 & + & + & 0 \\
$\quad$ Corno dorsal & 0 & 0 & 0 & 0 \\
Bulbo ao nível do óbex & & & & \\
Núcleo motor dorsal do vago & ++ & ++ & ++ & + \\
$\quad$ Núcleo ambíguo & 0 & + & ++ & + \\
Cerebelo / Ponte & & & & \\
$\quad$ Núcleos vestibulares & 0 & 0 & 0 & 0 \\
$\quad$ Formação reticular & 0 & ++ & + & + \\
Tálamo caudal ao corpo mamilar & & & & \\
$\quad$ Núcleo vermelho & +++ & +++ & ++ & + \\
Gânglio trigeminal & +++ & +++ & +++ & ++ \\
Gânglio celíaco & 0 & - & +++ & - \\
$\quad$ Gânglio espinhal & ++ & +++ & +++ & ++ \\
Gânglio estrelado & - & - & +++ & ++ \\
$\quad$ Nervo isquiádico & 0 & 0 & 0 & ++ \\
$\quad$ Nervo fibular & - & - & + & ++ \\
\hline
\end{tabular}

a Intensidade das lesões: (+) leve (1-5 neurônios cromatolíticos), (++) moderada (6-10 neurônios cromatolíticos), (+++) acentuada (mais que 10 neurônios cromatolíticos), (0) sem alterações, (-) não pesquisado.
Os principais achados histológicos foram lesões degenerativas e necróticas em neurônios dos cornos ventrais da medula espinhal, de núcleos específicos da medula oblonga, ponte, mesencéfalo e tálamo, além de gânglios nervosos espinhais, trigêmeos, estrelado e celíaco. As lesões eram bilaterais e relativamente simétricas. Os núcleos nervosos do tronco encefálico mais consistentemente afetados foram o vermelho, formação reticular da ponte, motor dorsal do nervo vago e ambíguo (Quadro 2). Não foram observadas lesões nos plexos mioentéricos e submucosais nos cortes de intestino dos animais deste estudo. Os núcleos oculomotor e hipoglosso estavam presentes e foram avaliados em todos os cortes do mesencéfalo e medula oblonga deste estudo, respectivamente e nestes não foram observados neurônios com lesões.

Os neurônios afetados apresentavam-se tumefeitos, arredondados, com citoplasma eosinofílico, homogêno e pálido, particularmente na porção central do pericário, e com grânulos de Nissl rarefeitos dispostos numa fina camada na periferia da célula ou praticamente ausentes. Os núcleos dos neurônios afetados, quando presentes no plano de corte do pericário, eram com freqüência periféricos e apresentavam-se como que rechaçados contra a membrana celular, com picnose ou cariorrexia (Fig.3A). Ocasionalmente estes neurônios cromatolíticos apresentavam vacúolos citoplasmáticos claros bem delimitados, de diferentes tamanhos que por vezes ocupavam boa parte do pericário. Na formação reticular da ponte do Bovino 5 alguns neurônios apresentavam citoplasma eosinofílico parcialmente fragmentado, margeado (satelitose) ou entremeado (neuronofagia) por células microgliais (Fig.3B), além de discretos aglomerados de células da glia e manguitos perivasculares linfocitário. Nos gânglios espinhais (Bovinos 2, 3 e 5), associados às lesões regressivas em neurônios, havia também fragmentação de neurônios com infiltração de macrófagos (neuronofagia), com aumento na celularidade ao redor de neurônios (satelitose) e preenchimento celular de locais antes ocupados por neurônios (provavelmente aglomerados de células satélites, os chamados nódulos de Nageotte).

Nos funículos dorsais da medula espinhal (Bovino 5) e nos nervos isquiádicos (Bovino 5) e fibulares (Bovinos 4 e 5) havia tumefação ou ausência de axônios, associados à dilatação da bainha de mielina (Fig.3C) e ocasionais macrófagos com citoplasma abundante e vacuolizado (câmaras de digestão). A imunomarcação de neurofilamento $\mathrm{H}$ associada ao azul rápido de luxol ressaltou os axônios tumefeitos e a conseqüente perda de mielina, respectivamente (Fig.3D). Os macrófagos associados às lesões axonais apresentaram no citoplasma marcação para neurofilamento e coloração pelo azul rápido de luxol. A imunomarcação do neurofilamento foi observada apenas em axônios do sistema nervoso central e nervos periféricos, não sendo notada marcação no pericário de neurônios cromatolíticos. Não foi evidenciado aumento na imunomarcação da GFAP em astrócitos das áreas contendo neurônios cromatolíticos. A imuno-histoquímica para 


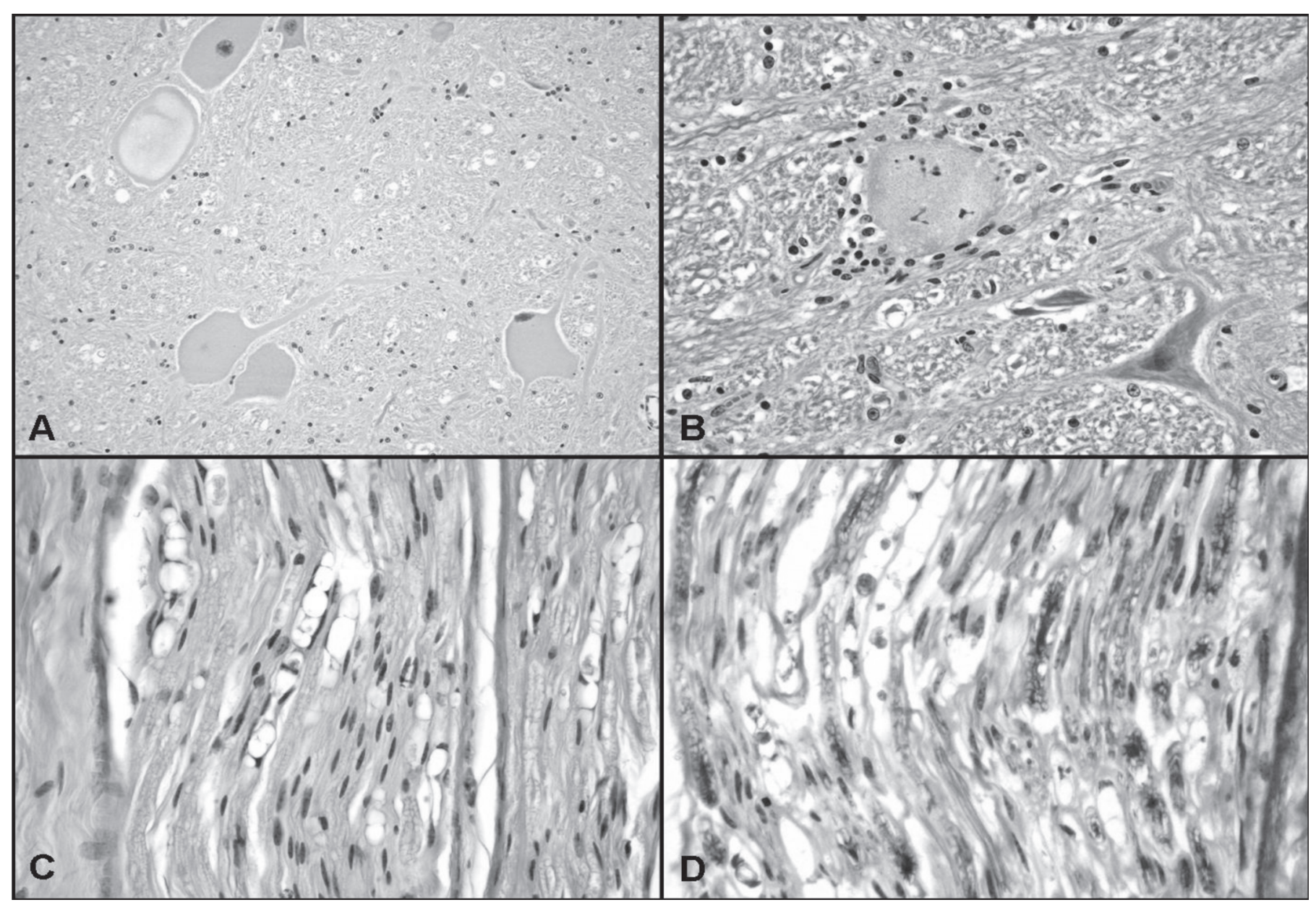

Fig.3. Neurotoxicose causada por Aspergillus clavatus em bovinos. (A) Bovino 5. Núcleo ambíguo. Neurônios tumefeitos com cromatólise total, aumento da eosinofilia citoplasmática, núcleos ausentes ou picnóticos e deslocados para periferia do pericário. Um neurônio apresenta marcada dissolução da porção central do pericário. HE, obj.20x. (B) Bovino 5. Formação reticular da ponte. Corpo do neurônio necrótico com marginação e infiltração (neuronofagia) de microglia. HE, obj.40x. (C e D) Bovino 5. Nervo fibular. (C) Tumefação, fragmentação e perda de axônios e vacuolização, indicando a perda de mielina. HE, obj.40x. (D) Tumefação e fragmentação de axônios demonstradas pela imunomarcação de neurofilamento (marrom escuro), com infiltração de macrófagos com citoplasma azulado, indicando a fagocitose de mielina (câmaras de digestão) nas áreas de perda de axônios. Azul rápido de luxol e imuno-histoquímica para neurofilamento. HE, obj.40x.

PrP nestes animais foi consistentemente negativa. As lesões musculares se caracterizavam na histopatologia por vacuolização intracitoplasmática, segmentos das miofibras tumefeitos, com aumento da eosinofilia e perda das estriações (necrose hialina) ou fragmentação (necrose flocular). No Bovino 2 e em uma amostra do Bovino 4 estas lesões foram acompanhadas de mineralização e/ ou fibrose e tentativas de regeneração. Mesmo animais sem lesões macroscópicas aparentes apresentavam lesões musculares em alguns músculos na microscopia, embora em geral leves. No Bovino 5 havia ainda degeneração Walleriana em parte dos ramos nervosos entremeados nas miofibras em alguns músculos avaliados (diafragma, longíssimo dorsal, subescapular e vasto medial). Não foram observadas lesões nas miofibras das áreas avaliadas do miocárdio, diafragma e músculos intercostais. Os demais órgãos avaliados não apresentavam alterações significativas.
Os fungos presentes nas amostras de bagaço de malte das duas propriedades e nos cultivos foram identificados como A. clavatus pelas características macro e micromorfológicas. Nas amostras da Propriedade 2, além deste, houve pequena contaminação por Rhizopus sp. As colônias de $A$. clavatus apresentavam aspecto granular, coloração azul esverdeada e, microscopicamente, conidióforos medindo 3-4 $\mu \mathrm{m}$ com típicas vesículas em forma de clava, inteiramente recobertas por fiálides curtos e conídios. A pesquisa de patulina foi negativa nas amostras de bagaço de malte da Propriedade 1. As temperaturas médias das propriedades nos períodos dos surtos foram de cerca de $21^{\circ} \mathrm{C}$ (Propriedade 1) e $26^{\circ} \mathrm{C}$ (Propriedade 2).

\section{DISCUSSÃO}

O diagnóstico da neurotoxicose causada por Aspergillus clavatus neste caso foi baseado no conjunto de dados epidemiológicos, clínicos, patológicos e micológicos. Os 
achados clínicos e patológicos foram semelhantes aos descritos em casos prévios naturais e experimentais de neurotoxicose associada à ingestão de alimentos contaminados por A. clavatus (Kellerman et al. 1976, 1984, Gilmour et al. 1989, Van der Lugt et al. 1994, 2002, Loretti et al. 2003, Colodel et al. 2004, El-Hage \& Lancaster 2004, McKenzie et al. 2004, Sabater-Vilar et al. 2004,).

Os sinais clínicos locomotores observados nos bovinos do presente estudo podem estar relacionados às lesões nos neurônios do tronco encefálico e medula espinhal (De Lahunta 1983, Barros et al. 2006, Chiocchetti et al. 2006). No entanto, A. clavatus pode produzir micotoxinas tremorgênicas que podem causar sinais semelhantes e desta forma contribuir para manifestação clínica da doença (Kellerman et al. 1976, 1984, Varga et al. 2003, Sabater-Vilar et al. 2004).

A degeneração e necrose neuronal cromatolítica observadas nos casos presentes são semelhantes às descritas anteriormente (Kellerman et al. 1976, 1984, Gilmour et al. 1989, Van der Lugt et al. 1994, 2002, Loretti et al. 2003, Colodel et al. 2004, El-Hage \& Lancaster 2004, McKenzie et al. 2004, Sabater-Vilar et al. 2004). O envolvimento de neurônios dos gânglios trigêmeo, estrelado e celíaco descrito nesse estudo não tem sido relatado em outros surtos relacionados a $A$. clavatus (El-Hage \& Lancaster 2004). Devido ao envolvimento, nesses bovinos, de neurônios motores superiores e inferiores, sensórios e de gânglios autônomos, sugere-se que seja mais apropriado classificar a enfermidade como uma doença neuronal degenerativa multissistêmica. Os neurônios motores superiores dos núcleos vermelhos (Chiocchetti et al. 2006) e formação reticular da ponte (De Lahunta, 1983) têm longas projeções axonais caudais para medula espinhal e foram afetados nesses casos. Entretanto, o córtex motor, geralmente afetado em doenças dos neurônios motores de humanos, era poupado nos bovinos aqui apresentados, conforme observado anteriormente (Kellerman et al. 1976, 1984, Loretti et al. 2003, McKenzie et al. 2004). De forma semelhante ao sugerido na doença do neurônio motor de eqüinos (Cummings et al. 1990), o não envolvimento do córtex motor na neurotoxicose causada pelo $A$. clavatus em bovinos pode estar associado ao desenvolvimento rudimentar do trato cortico-espinhal nesta espécie (De Lahunta 1983, Chiocchetti et al. 2006). Outros grupos neuronais afetados, como neurônios motores inferiores e dos gânglios espinhais, também emitem longas projeções axonais (De Lahunta et al. 1983), o que indica que essa característica seja importante na susceptibilidade dos neurônios nesta doença. A imuno-histoquímica não evidenciou acúmulo de neurofilamentos no pericário de neurônios afetados, comoé observado em algumas doenças de neurônios motores (Cummings et al. 1990, Summer et al. 1994). Nos casos aqui apresentados, também não foram observadas alterações em astrócitos ou vasos nas áreas com lesões neuronais, como descrito nas neuropatias tóxicas associadas à privação de energia (Cavanagh 1993).
Como ocorrido anteriormente (Kellerman et al. 1976), a participação da patulina na doença descrita aqui não pode ser demonstrada. A participação desta e de outras micotoxinas na doença associada ao $A$. clavatus ainda necessita de comprovação (Lopez-Dias \& Flannigan 1997, McKenzie et al. 2004, Sabater-Vilar et al. 2004).

Lesões axonais similares às observadas nos casos mais crônicos deste estudo têm sido atribuídas a axonopatia (Gilmour et al. 1989, Van Der Lugt et al. 1994, Sabater-Vilar et al. 2004), mas podem também estar relacionadas a agressões subletais no pericário de neurônios (Cummings et al. 1990). Lesões com morfologia e distribuição semelhantes às descritas ocorrem na axonopatia distal ou "dying back" (Cavanagh 1979). Este mecanismo já foi proposto em um surto associado a A. clavatus (Van Der Lugt et al. 1994). Em neuropatias tóxicas, alterações no pericário podem surgir nas fases iniciais da doença, quando os axônios estão praticamente intactos (Sterman 1983), o que talvez possa explicar a ausência de lesões axonais na maioria dos bovinos do presente estudo. A ausência de lesões em axônios da medula espinhal (Loretti et al. 2003, McKenzie et al. 2004) ou de nervos periféricos (El-Hage \& Lancaster 2004) tem sido descrita também em outros surtos.

Vacúolos de diferentes tamanhos e bem definidos, por vezes ocupando boa parte do citoplasma dos neurônios, foram ocasionalmente observados em neurônios sem outras alterações evidentes nos gânglios espinhais e trigeminal, além do núcleo vermelho de alguns animais. Vacúolos semelhantes a estes têm sido descritos ao menos no núcleo vermelho (Gavier-Widen et al. 2001) e no gânglio trigeminal (Rech et al. 2006), de animais sem manifestações de sinais neurológicos o que compromete a inclusão destes no quadro patológico associado a $A$. clavatus. A vacuolização da substância branca observada em um bovino do presente surto, restrita apenas a substância nigra, é semelhante à relatada em animais sem sinais de doença neurológica (Gavier-Widen et al. 2001) e dessa forma não foi relacionada ao quadro associado a A. clavatus.

Como descrito em outros surtos havia degeneração e necrose em músculos esqueléticos dos membros (Kellerman et al. 1976, Van Der Lugt et al. 2002, Loretti et al. 2003, Colodel et al. 2004), por vezes acompanhadas de mineralização distrófica (Loretti et al. 2003). Estas lesões podem, entretanto, estar ausentes em alguns surtos (Kellerman et al. 1984, Gilmour et al. 1989, Van Der Lugt et al. 1994, El-Hage \& Lancaster 2004, McKenzie et al. 2004). A patogênese das lesões musculares ainda é indeterminada (Gilmour et al. 1989, Loretti et al. 2003, ElHage \& Lancaster 2004).

Conforme relatado, após a remoção do alimento contaminado, tanto pode haver recuperação clínica de animais com sinais neurológicos pronunciados (Kellerman et al. 1984, Gilmour et al. 1989, Loretti et al. 2003, ElHage \& Lancaster 2004), quanto a ocorrência de casos novos (Gilmour et al. 1989, El-Hage \& Lancaster 2004). 
O diagnóstico diferencial para a doença causada por A. clavatus deve incluir principalmente as síndromes tremorgênicas, o botulismo, a raiva e a leucose com envolvimento da medula espinhal. As lesões musculares, quando presentes, são de grande auxílio na diferenciação dessas enfermidades. Quando há lesões musculares, o diagnóstico diferencial deve incluir miopatias tóxicas e nutricionais, nestes casos, porém não há sinais neurológicos (Loretti et al. 2003, Barros et al. 2006, Riet-Correa et al. 2007). Apesar das semelhanças clínicas, em geral, nas intoxicações por Claviceps paspali, Stenocarpella (Diplodia) maydis, Cynodon dactylon, Lolium perenne e Ipomoea asarifolia não são descritas lesões macro ou microscópicas(Loretti et al. 2003, Barros et al. 2006, RietCorrea et al. 2007). Nas intoxicações por plantas que causam doenças do armazenamento lisossomal em bovinos (Sida carpinifolia, Solanum fastigiatum, Ipomoea carnea subsp. fistulosa e Turbina cordata), a doença tremorgênica é acompanhada por vacuolização citoplasmática em diversos tecidos, incluindo neurônios, particularmente os do córtex cerebelar e cerebral, tálamo, mesencéfalo e medula espinhal. Embora presente na doença causada por $A$. clavatus a vacuolização neuronal não tem sido observada no córtex cerebelar ou cerebral. Na intoxicação por Phalaris spp., áreas verde azuladas no encéfalo (tálamo, mesencéfalo e medula oblonga) observadas na macroscopia correspondem a pigmento marrom-amarelado no citoplasma de neurônios semelhante a lipofucsina. As outras doenças mencionadas podem ser diferenciadas por lesões distintas (leucose e raiva) e ausência de lesões (botulismo) ou por dados epidemiológicos (intoxicação tardia por organofosforados) (Loretti et al. 2003, Barros et al. 2006, Riet-Correa et al. 2007). A encefalopatia espongiforme bovina (EEB) também é um importante diferencial devido às semelhanças clínicas e histológicas. Nestes casos a imuno-histoquímica é crucial para uma diferenciação inequívoca (Loretti et al. 2003, Barros et al. 2006, Riet-Correa et al. 2007). Nos casos aqui apresentados a imuno-histoquímica utilizando o anticorpo monoclonal anti-proteína príon nos cortes da medula oblonga ao nível do óbex de todos os animais foi consistentemente negativa.

A proliferação de diversos tipos de fungos ambientais pode ocorrer desde a germinação do grão de cevada, durante a produção do malte, até a utilização do subproduto nas propriedades rurais (Lopez-Diaz \& Flannigan 1997, Simas et al. 2007). O bagaço de malte tem uso bastante difundido em rebanhos leiteiros na região (Barros et al. 2006) e a grande maioria de produtores de leite, inclusive vizinhos das propriedades que foram afetadas e que recebiam o mesmo material, não registrou problemas. Condições importantes para crescimento e produção de micotoxinas por fungos, como temperatura ambiente, substrato rico em nutrientes e umidade foram propícias ao desenvolvimento do fungo (Lopez-Dias \& Flannigan 1997, McKenzie et al. 2004). Assim, a presença de cepas toxigênicas de $A$. clavatus associada a fatores de arma- zenagem e manejo do bagaço de malte pode ter sido de importância para o surgimento do problema.

Níveis menores de contaminação fúngica têm sido associados com o consumo do bagaço de malte em um curto período de tempo e estocagem com boa drenagem (Simas et al. 2007). Neste sentido, a oferta do subproduto na forma dessecada tem sido preconizada como mais segura (Barros et al. 2006).

Agradecimentos.- Este estudo teve suporte financeiro do Conselho Nacional de Desenvolvimento Científico e Tecnológico (CNPq) e da Coordenação de Aperfeiçoamento de Pessoal de Nível Superior (CAPES). Os autores agradecem ao professor Cláudio Estevão Farias da Cruz (UFRGS) pela revisão deste artigo e ao professor Marcos Dutra Duarte (UFPA) pelo auxílio na interpretação dos sinais clínicos.

\section{REFERÊNCIAS}

Barros C.S.L., Driemeier D., Dutra I.S. \& Lemos R.A.A. 2006. Doenças do Sistema Nervoso de Bovinos no Brasil. AGNS Gráfica e Editora, São Paulo. 207p.

Cavanagh J.B. 1979. The 'dying back' process. Arch. Pathol. Lab. Med. 103: 659-664.

Cavanagh J.B. 1993. Selective vulnerability in acute energy deprivation syndromes. Neuropathol. Appl. Neurobiol. 19:461-470.

Chiocchetti R., Bombardi C., Grandis A., Mazzuoli G., Gentile A., Pisoni L., Joechler M. \& Lucchi M.L. 2006. Cytoarchiteture, morphology, and lumbosacral spinal cord projections of the red nucleus in cattle. Am. J. Vet. Res. 67(10):1662-1669.

Colodel E.M., Schmitz M., Traverso S.D., Sanches E.M.C., Ferreiro F., Loretti A.P., Correa A.M.C., Seitz A.L. \& Driemeier D. 2004. Aspectos clínicos e patológicos da doença neurológica de bovinos reproduzida pela administração de milho contaminado com Aspergillus clavatus. Pesq. Vet. Bras. 24(Supl.):16-17.

Cummings J.F., De Lahunta A., George C., Fuhrer L., Valentine B.A. Cooper, B.J. Summers B.A., Huxtable C.R. \& Mohammed H.O. 1990. Equine motor neuron disease: A preliminary report. Cornell Vet. 80(4):357-379.

De Lahunta A. 1983. Veterinary Neuroanatomy and Clinical Neurology. $2^{\text {nd }}$ ed. W.B. Saunders Company, London. 471p.

El-Hage C.M. \& Lancaster M.J. 2004. Mycotoxic nervous disease in cattle fed sprouted barley contaminated with Aspergillus clavatus. Aust. Vet. J. 82(10):639-641.

Gavier-Widen D., Wells A.H., Simmons M.M., Wilesmith J.W.W. \& Ryan J. 2001. Histological observations on the brains of symptomless 7year-old cattle. J. Comp. Pathol. 124:52-59.

Gilmour J.S., Inglis D.M., Robb J. \& Maclean M.A 1989. A fooder mycotoxicosis of ruminants caused by contamination of distillery byproduct with Aspergillus clavatus. Vet. Rec. 124:133-135.

Kellerman T.S., Pienaar J.G., Van der Westhuizen G.C.A., Anderson L.A.P. \& Naudé T.W. 1976. A highly fatal tremorgenic mycotoxicosis of cattle caused by Aspergillus clavatus. Onderstepoort J. Vet. Res. 43(3):147-154.

Kellerman T.S., Newsholme S.J., Coetzer J.A.W. \& Van der Westhuizen G.C.A. 1984. A tremorgenic mycotoxicosis of cattle caused by maize sprouts infested with Aspergillus clavatus. Onderstepoort J. Vet. Res. 51:271-274.

Kellerman T.S., Coetzer J.A.W., Naudé T.W. \& Botha C.J. 2005. Plant Poisonings and Mycotoxicosis of Livestock in Southern Africa. $2^{\text {nd }}$ ed. Oxford University Press, Cape Town, p.63-113.

Lopez-Diaz T. \& Flannigan B. 1997. Production of patulin and cytochalasin E by Aspergillus clavatus during malting of barley and wheat. Int. J. Food Microbiol. 35:129-136.

Loretti A.P., Colodel E.M., Driemeier D., Corrêa A., Bangel Jr J.J. \& 
Ferreiro L. 2003. Neurological disorder in dairy cattle associated with consumption of beer residues contaminated with Aspergillus clavatus. J. Vet. Diagn. Invest. 15:123-132.

McKenzie R.A., Kelly M.A., Shivas R.G., Gibson J.A., Cook P.J., Widderick K. \& Guilfoyle A.F. 2004. Aspergillus clavatus tremorgenic neurotoxicosis in cattle fed sprouted grains. Aust. Vet. J. 82(10):635638.

Prophet E.B., Mills B., Arrington J.B., Leslie H. \& Sobin L.H.M.D. 1992. AFIP Laboratory Methods in Histotechnology. The Registry of Pathology, Washington, DC.

Riet-Correa F., Schild A.L., Lemos R.A. \& Borges J.R. 2007. Doenças de Ruminantes e Eqüídeos. Vol.2. 3 ${ }^{\underline{a}}$ ed. Pallotti, Santa Maria. 694p.

Rech R.R., Rissi D.R., Silva M.C., Inkelmann M.A. \& Barros C.S.L. 2006. Histomorfologia do gânglio de Gasser, da rete mirabile carotídea e da hipófise de bovinos: estudo de 199 casos. Pesq. Vet. Bras. 26(2):105-111.

Sabater-Vilar M., Maas R.F.M., De Bosschere H., Ducatelle R. \& FinkGremmels J. 2004. Patulin produced by an Aspergillus clavatus isolated from feed containing malting residues associated with a lethal neurotoxicosis in cattle. Mycopathologia 158:419-426.

Santos M.S. \& Ribeiro F.M. 2005. Cervejas e Refrigerantes. CETESB, São Paulo. 58p.
Simas M.M.S., Botura M.B., Correa B. Sabino M., Mallmann C.A., Bitencourt C.B.S.C. \& Batatinha M.J.M. 2007. Determination of fungal microbiota and mycotoxins in brewers grain used in dairy cattle feeding in the State of Bahia, Brazil. Food Control 18:404-408.

Sterman A.B. 1983. Altered sensory ganglia in acrilamide neuropathy. J. Neuropathol. Exp. Neurology 42(2):166-176.

Van der Lugt J.J., Kellerman T.S., Van Vollenhoven A. \& Nel P.W. 1994. Spinal cord degeneration in adult dairy cows associated with the feeding of sorghum beer residues. J. South Afr. Vet. Assoc. 65(4):184188.

Van der Lugt J.J., Prozesky L. \& Van Wilpe E. 2002. Acute Aspergillus clavatus poisoning in cattle: Light microscopical and ultrastructural lesions in the spinal cord, p.131-141. In: Van der Lugt J.J. (Ed.), The Clinicopathology and Pathology of Selective Toxicoses and Storage Diseases of the Nervous System of Ruminants in Southern Africa. Doctoral Thesis, University of Utrecht, Netherlands.

Varga J., Rigó K., Molnár J., Tóth B., Szencz S., Téren J. \& Kozakiewicz Z. 2003. Mycotoxin production and evolutionary relationships among species of Aspergillus section Clavati. Antonie van Leeuwenhoek 83:191-200.

Yoshikawa T. 1968. Atlas of the Brains of Domestic Animals. University of Tokyo Press, Tokyo. 